\title{
Potencial evocado miogênico vestibular (VEMP)
}

\author{
Alcione B. Pereira, ${ }^{1 *}$ Gabriela S. M. Silva, ${ }^{1}$ Lilian Felipe, ${ }^{2}$ Aída R. M. Assunção, ${ }^{3}$ Ciríaco C. T. Atherino ${ }^{3}$
}

\begin{abstract}
Resumo
O objetivo desta revisão é destacar a história, método de realização do teste do potencial evocado miogênico vestibular (VEMP), além de discutir as suas aplicações, no intuito de ajudar os profissionais no manejo desse novo recurso. O VEMP vem sendo empregado como exame complementar em estudos otoneurológicos, para avaliação funcional das vias envolvidas desde a condução do estímulo na orelha média até a resposta reflexa no músculo esternocleidomastóideo. O registro do VEMP pode ser obtido a partir de um aparelho de potenciais evocados auditivos. A estimulação auditiva com sons de elevada intensidade é a técnica mais utilizada. Estudos realizados verificaram o valor do VEMP no diagnóstico e monitoramento de diversas doenças de origem central e periférica, como doença de Meniere, trauma acústico, fenômeno de Tullio, degeneração espinocerebelar, deiscência do canal vestibular superior, neurinoma do acústico, esclerose múltipla, vertigem posicional paroxística benigna e neuronite vestibular.
\end{abstract}

Descritores: Potencial evocado motor; Nervo vestibular; Testes de função vestibular.

\section{Abstract \\ Vestibular evoked myogenic potential (VEMP)}

The aim of this review is to discuss the Vestibular Evoked Myogenic Potential (VEMP) - its history, methods, and potential uses. VEMP has been used for otoneurological studies, as it allows the functional assessment of pathways involved since the stimulus conduction in the middle ear to the reflex response of the sternocleidomastoid muscle. VEMP records can be obtained using an auditory evoked potentials device. Auditory stimulation with high intensity sounds is the most used technique. The value of VEMP has been demonstrated for diagnosis and monitoring of various diseases of central or peripheral origin, such as Meniere's disease, acoustic trauma, Tullio phenomenon, spinocerebellar degeneration, dehiscence of the superior vestibular canal, acoustic neuroma, multiple sclerosis, benign paroxysmal positional vertigo, and vestibular neuronitis.

Keywords: Evoked potentials, motor; Vestibular nerve; Vestibular function tests.
1. Departamento de Fonoaudiologia. Universidade Veiga de Almeida. Rio de Janeiro, RJ, Brasil.

2. Mestrado Profissional em Fonoaudiologia. Programa de PósGraduação em Fonoaudiologia. Universidade Veiga de Almeida. Rio de Janeiro, RJ, Brasil.

3. Departamento de Especialidades Cirúrgicas.Faculdade de Ciências Médicas. Universidade do Estado do Rio de Janeiro. Rio de Janeiro, RJ, Brasil.

*Endereço para correspondência:

Av. Alfredo Balena, 189/1702

Belo Horizonte, MG, Brasil. CEP: 30130-100.

E-mail: alcipere@terra.com.br

Revista HUPE, Rio de Janeiro, 2015;14(1):56-59

doi: $10.12957 /$ rhupe.2015.16210

Recebido em 28/07/2014. Aprovado em 01/10/2014.

\section{Resumen}

\section{Potencial vestibular miogénico evocado (PVME)}

El objetivo de esta revisión es destacar la historia y el método de realización de la prueba del Potencial Vestibular Miogénico Evocado (PVME) y discutir sus aplicaciones, con el objetivo de ayudar a los profesionales en el manejo de ese nuevo recurso. El PVME viene siendo empleado como examen complementario en estudios otoneurológicos para evaluación funcional de las vías implicadas desde la conducción del estímulo en el oído medio hasta la respuesta refleja en el músculo esternocleidomastoideo. El registro del PVME puede ser obtenido a partir de un aparato de potenciales evocados auditivos. La estimulación auditiva con sonidos de alta intensidad es la técnica más utilizada. Los estudios realizados verificaron el valor del PVME en el diagnóstico y monitoreo de diversas enfermedades de origen central y periférico, como enfermedad de Meniere, trauma acústico, fenómeno de Tullio, degeneración espinocerebelosa, dehiscencia del canal vestibular superior, neurinoma del acústico, esclerosis múltiple, vértigo posicional paroxístico benigno y neuronitis vestibular.

Palabras clave: Potenciales evocados motores; Nervio vestibular; Pruebas de función vestibular. 


\section{Introdução}

O potencial evocado miogênico vestibular (VEMP) vem sendo empregado como exame complementar em estudos otoneurológicos. São potenciais evocados de longa latência que consistem em respostas miogênicas originadas por estimulação sonora que ativa a mácula sacular, o nervo vestibular inferior e as vias vestíbulo espinhais descendentes. ${ }^{1}$ Essas respostas são registradas por eletromiografia de superfície sobre a musculatura cervical. O VEMP é um exame que tem sido amplamente estudado.

Vários estudos descrevem que o VEMP apresenta várias características favoráveis à sua utilização, como o fato de ser um exame objetivo, confiável, não invasivo, de baixo custo, de fácil execução, rápido e sem desconforto para o paciente. ${ }^{2-4}$

O potencial evocado miogênico vestibular - vestibular evoked myogenic potential - (VEMP) avalia a resposta muscular decorrente de estimulação auditiva. Essa resposta neural é um arcorreflexo de três neurônios que envolvem a orelha interna, o tronco encefálico e a via vestibuloespinhal. Esse arcorreflexo está envolvido na manutenção do equilíbrio corporal. Desse modo, enfermidades que interfiram na condução neural desde a orelha interna, passando pelo tronco encefálico, pelo trato espinhal anterior e pelo segundo neurônio motor, podem interferir na resposta.

Como princípio básico da avaliação de qualquer potencial evocado, mede-se o tempo entre o estímulo e a resposta, classificando-o como normal ou alterado a partir do tempo de duração e da morfologia das ondas elétricas geradas. A importância do VEMP está relacionada à avaliação funcional das vias envolvidas na condução do estímulo da orelha interna à resposta muscular reflexa. A vantagem desse exame é que alterações ainda não detectadas clinicamente ou visíveis por exames de imagem poderiam ser percebidas precocemente pelo VEMP. O que ainda não se sabe é a validade do VEMP para esse fim.

Atualmente, o VEMP vem sendo estudado para determinar a presença de alterações na orelha interna ou na condução neural, desde o nervo vestibular inferior até a resposta motora muscular.

O objetivo desta revisão é destacar a história, método de realização do teste do potencial evocado miogênico vestibular, além de discutir as suas aplicações, no intuito de ajudar os profissionais no manejo desse novo recurso.

\section{História}

Colebatch e colaboradores, em 1992, ${ }^{5}$ estudaram pela primeira vez o VEMP no músculo esternocleidomastóideo. Esse estudo menciona que o primeiro registro desse reflexo no músculo foi realizado em 1964, por Bickford. ${ }^{6}$ Colebatch e colaboradores em $1994^{7}$ verificaram que o reflexo é dependente da integridade do sistema otolítico vestibular, sendo as primeiras evidências da possibilidade de se usar o exame para diagnóstico de afecções no sistema vestibular.

Em 1994, Halmagyi e colaboradores ${ }^{4}$ descreveram $^{-}$ três testes para o diagnóstico de patologias neste sistema, dentre os quais o VEMP. Descreveram ainda a possibilidade de realização do VEMP para diferenciar as alterações vestibulares lateralmente. Atualmente ele vem sendo utilizado para avaliação funcional das vias envolvidas desde a condução do estímulo na orelha média até a resposta reflexa no músculo esternocleidomastóideo.

\section{Método de realização}

O VEMP pode ser obtido a partir de um aparelho de potenciais evocados auditivos. Podem ser eliciados por estímulos acústicos, elétricos ou mecânicos. A estimulação auditiva com sons de elevada intensidade é a técnica mais utilizada. ${ }^{8}$

Com relação aos parâmetros para realização do VEMP, alguns estudos utilizaram cliques para estimulação da mácula., ${ }^{7,9}$ Dentre todos os órgãos vestibulares, o sáculo, situado exatamente abaixo da base do estribo, é o mais sensível ao som. ${ }^{1} \mathrm{O}$ reflexo se origina no sáculo, é transmitido para os neurônios do Gânglio de Scarpa, caminha pelo nervo vestibular inferior, núcleo vestibular e trato vestibuloespinhal e chega aos neurônios motores do músculo. ${ }^{4,10}$

Quanto à colocação dos eletrodos, tem-se observado na literatura que o eletrodo de superfície é colocado geralmente sobre o músculo esternocleidomastoideo ${ }^{5} \mathrm{e}$ quanto à intensidade dos estímulos, trabalhos clínicos observaram que o limiar de excitabilidade da mácula sacular é em torno de 80 dB NPS. ${ }^{3}$

Para ativação do músculo esternocleidomastóideo, durante a realização do exame, alguns autores recomendam que o indivíduo permaneça sentado, fazendo pressão com a testa sobre uma barra posicionada à frente da cabeça, enquanto outros prescrevem que ele deve permanecer em decúbito dorsal horizontal, elevando a cabeça. Nas duas situações, obtém-se ativação simultânea de ambos os músculos. Todavia, avaliou-se que 
a posição que promove a melhor ativação muscular é a rotação lateral máxima da cabeça, com o indivíduo sentado. ${ }^{11-12}$

Com a realização do exame, é obtido um traçado constituído por dois complexos de ondas bifásicas. $\mathrm{O}$ primeiro potencial apresenta pico positivo (P) com latência média de 13 milisegundos (ms), seguido de pico negativo $(\mathrm{N})$ com latência média de $23 \mathrm{~ms} .{ }^{12}$ Como princípio básico da avaliação de qualquer potencial evocado, mede-se o tempo entre o estímulo e a resposta, classificando-o como normal ou alterado a partir do

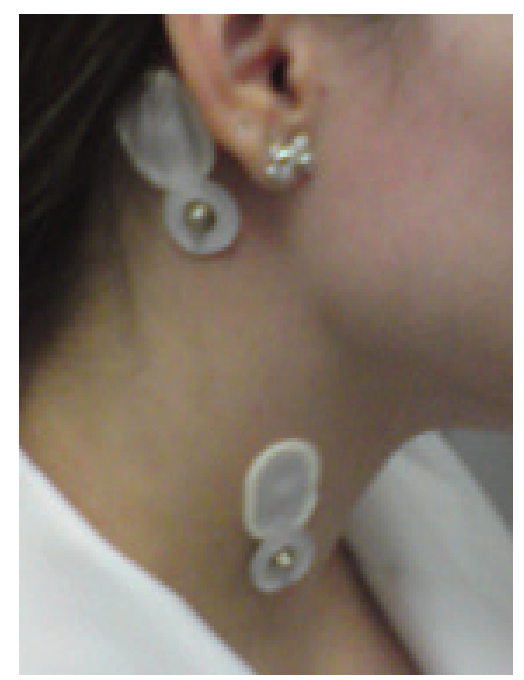

Figura 1. Eletrodo no terço médio do músculo esternocleidomastoídeo. tempo que transcorre entre a estimulação até o aparecimento das ondas. ${ }^{8} \mathrm{O}$ aumento da latência dos picos, no VEMP, está associado à alteração da velocidade de condução neuronal ${ }^{13}$

A amplitude de resposta reflete a magnitude do reflexo muscular, no entanto existe variação interpessoal de massa e tônus da musculatura. Assim, utiliza-se como variável analisada referente à amplitude o índice de assimetria. Esse é calculado pela diferença interaural da amplitude da resposta, ponderada pela média da resposta de cada paciente.

\section{Aplicações clínicas}

O sistema vestibular é bem mais complexo e menos conhecido e estudado que o sistema auditivo, por isso o interesse pelo potencial evocado miogênico vestibular (VEMP) cresceu nos últimos anos, em virtude desses potenciais refletirem a função de órgãos otolíticos (sáculo) e do nervo vestibular inferior (NVI), algo até então não possível de ser avaliado pelos diversos outros testes vestibulares, como a vectoeletronistagmografia, a prova da cadeira rotatória e a prova da plataforma. ${ }^{15}$

As pesquisas relacionadas à aplicação clínica do VEMP expandiram-se devido à necessidade de definir a acuidade desse exame. Assim, estudos têm proposto que o VEMP seja utilizado como exame complementar na avaliação otoneurológica atualmente utilizada.

Estudos realizados verificaram o valor do VEMP no diagnóstico e monitoramento de diversas doenças de origem central e periférica: doença de Meniere, ${ }^{16-21}$

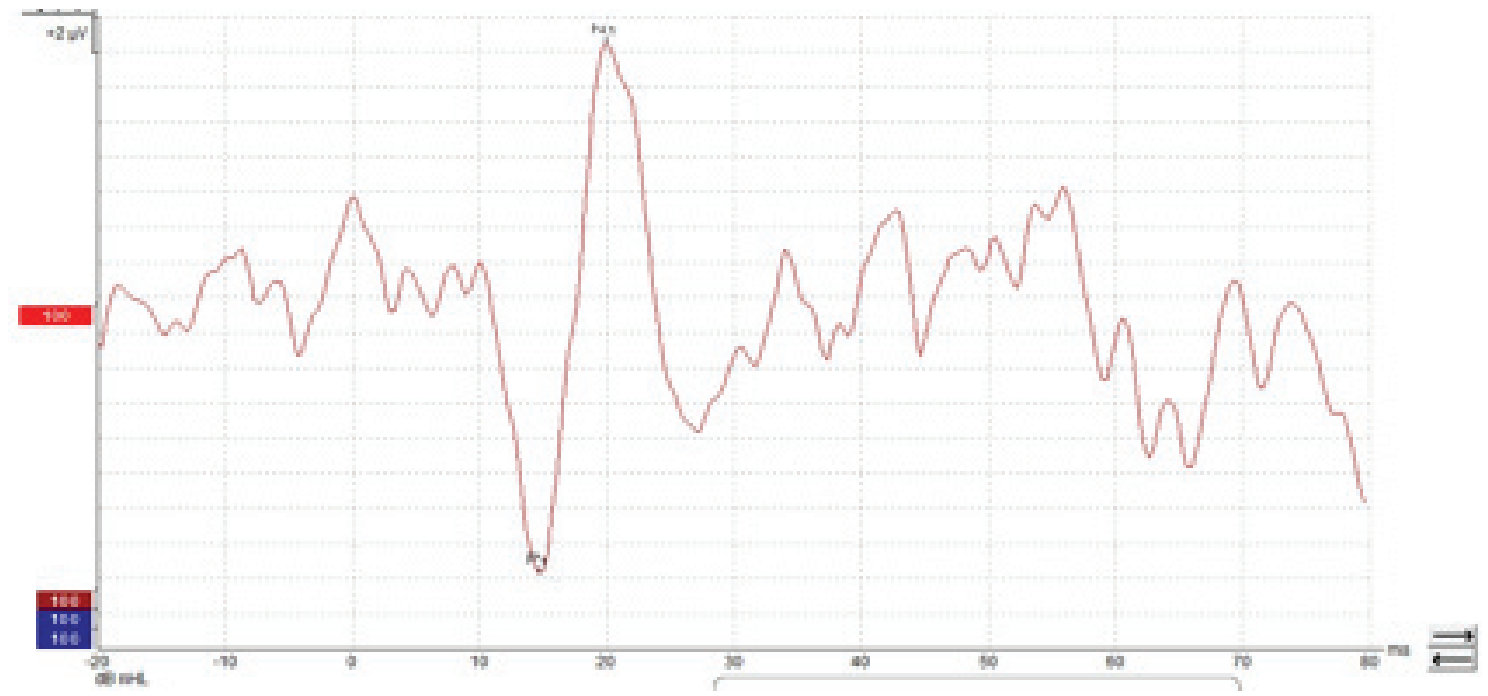

Figura 2. Traçado obtido com aparelho Eclipse em resposta a um "click". Observem as respostas P1 (P13) e N1 (N23). 
trauma acústico, ${ }^{22}$ fenômeno de Tullio, ${ }^{7}$ degeneração espinocerebelar, ${ }^{23}$ deiscência do canal vestibular superior, ${ }^{24}$ neurinoma do acústico, ${ }^{11}$ esclerose múltipla, $, 10,25-26$ vertigem posicional paroxística benigna, ${ }^{27}$ neuronite vestibular, ${ }^{28}$ entre outras.

\section{Conclusões}

O teste de VEMP é um novo exame complementar, que pode contribuir, juntamente com outras provas otoneurológicas, para o diagnóstico das mais diversas patologias vestibulares, mostrando ser um exame promissor, principalmente por nos fornecer informações sobre a função do sáculo e da divisão inferior do nervo vestibular. Além do mais, tem várias características favoráveis à sua utilização, como o fato de ser um exame objetivo, confiável, não invasivo, de baixo custo, de fácil execução, rápido e sem desconforto para o paciente. Vale a pena ressaltar, a necessidade de uma padronização do exame, não só na população adulta como também na população pediátrica.

\section{Referências}

1. Herdman, SJ. Potenciais evocados miogênicos vestibulares: um teste da função sacular In: Reabilitação vestibular. Barueri, SP: Manole; 2002. 201-211p.

2. David R, Colafemina JF. Potenciais miogênicos evocados vestibulares (VEMP): uma revisão bibliográfica. Rev. Bras. Otorrinolaringol. 2002;68(1):113-117.

3. Felipe L, Santos MAR, Gonçalves DU. Potencial evocado miogênico vestibular (Vemp): avaliação das respostas em indivíduos normais. Pró-FonoRevista de atualização científica. 2008;20(4):249-54.

4. Halmagly GM, Colebatch JG, Curthoys IS. New tests of vestibular function. Baillière's Clinical Neurology. 1994;3(3):485-500.

5. Colebatch JG, Halmagly GM. Vestibular evoked potentials in human neck muscles before and after unilateral vestibular deafferentation. Neurology. 1992;42(8):1635-1636.

6. Bickford RG, Jacobson JL, Cody DT. Nature of average evoked potentials to sound and other stimuli in man. Ann N Y Acad Sci. 1964;112:204-23.

7. Colebatch JG, Rothwell JC, Bronstein A, et al.. Click-evoked vestibular activation in the Tullio phenomenon. J Neurol Neurosurg Psychiatry. 1994;57(12):1538-1540.

8. Rosegren SM. Welgampola MS, Colebatch JG. Vestibular evoked myogenic potentials: past, present and future. Clin Neurophysiol. 2010;121(5):636-51.

9. Aidar RC, Suzuki FA. Potencial evocado miogênico vestibular: novas perspectivas diagnósticas em esclerose múltipla. Rev Bras Otorrinolaringol. 2005;71(1):48-54.
10. Murofushi T, Shimizu K, Takegoshi H. Diagnostic value of prolonged latencies in the vestibular evoked myogenic potential. Arch Otolaryngol Head Neck Surg. 2001;127(9):1069-72.

11. Murofushi T, Matsuzaki M, Mizuno M. Vestibular evoked myogenic potentials in patients with acoustic neuromas. Arch Otolaryngol Head Neck Surg. 1998;124(5):509-12.

12. $\mathrm{Wu} \mathrm{CH}$, Young $\mathrm{YH}$, Murofushi T. Tone burst evoked myogenic potentials in human neck flexor and extensor. Acta Otolaryngol. 1999;119:119:741-744.

13. Almeida RR. Potenciais evocados miogênicos vestibulares: estudo em indivíduos normais. 1999. Tese - Faculdade de Medicina da Universidade de São Paulo, São Paulo, 1999.

14. Ribeiro S, Almeida RR, Caovilla HH, et al. Dos potenciais evocados miogênicos vestibulares nas orelhas comprometida e assintomática na Doença de Meniere unilateral. Rev. Bras. Otorrinolaringol. 2005;71(1): 60-66.

15. Rauch SD. Vestibular evoked myogenic potentials. Curr Opin Otolaryngol Head Neck Surg. 2006;14(5):299-304.

16. Kuo SW, Yang TH, Young YH. Changes in vestibular evoked myogenic potentials after Meniere attacks. Ann Otol Rhinol Laryngol. 2005;114(9):717-21.

17. Lin MY, Timmer FC, Oriel BS, et al. Vestibular evoked myogenic potentials (VEMP) can detect asymptomatic saccular hydrops. Laryngoscope. 2006;116(6):987-92.

18. Magliulo G, Gianfone G, Gagliardi M, et al. Vestibular evoked myogenic potentials and glycerol testing. Laryngoscope. 2004;114(2):338-43.

19. Rauch SD, Zhou G, Kujawa SG, et al. Vestibular evoked myogenic potentials show altered tuning in patients with Meniere's disease. Otol Neurotol. 2004;25(3):333-8.

20. Timmer FC, Zhou G, Guinan JJ, et al. Vestibular evoked myogenic potentials (VEMP) in patients with Meniere's disease with drop attacks. Laryngoscope. 2006;116(5):776-9.

21. De Waele C, Huy PT, Diard JP, et al. Saccular dysfunction in Meniere's disease. Am J Otol. 1999;20(2):223-32.

22. Wang CT, Young YH. Comparison of the head elevation and rotation methods in eliciting vestibular evoked myogenic potentials. Ear Hear. 2006, 27:376-381.

23. Takegosh H, Murofushi T. Vestibular evoked myogenic potentials in patients with spinocerebellar degeneration. Acta Otolaryngol. 2000;120(7)821-24.

24. Brantberg K, Bergenius J, Tribukait A. Vestibular evoked myogenic potentials in patients with dehiscence of the superior semicircular canal. Acta Otolaryngol. 1999;119(6):633-40.

25. Alpini D, Pugnetti L, Caputo D, et al. Vestibular Evoked Myogenic Potentials in Multiple Sclerosis: A Comparison Between Onset and Definite Cases. Int Tinnitus J. 2005;11(1):48-51.

26. Versino M, Colnaghi S, Callieco R, et al. Vestibular evoked myogenic potentials in multiple sclerosis patients. Clin. Neurophysiol. 2002;113:1464-1469.

27. Akkuzu G, Akkuzu B, Ozluoglu LN. Vestibular evoked myogenic potentials in benign paroxysmal positional vertigo and Meniere's disease. Journal Eur Arch Otorhinolaryngol. 2006;263(6):510-7.

28. Halmagyi GM, Aw ST, Karlberg M, et al. Inferior vestibular neuritis. Ann N Y Acad Sci. 2002;956:306-13. 